\title{
La Administración por Valores, como modelo de implementación en la Facultad de Ciencias Administrativas y Contables de la Universidad de La Salle, Bogotá - Colombia.
}

\author{
Andrés Felipe Sánchez Díez \\ Universidad de La Salle
}

Recibido: 7 de febrero de 2013

Aceptado: 3 de mayo de 2013

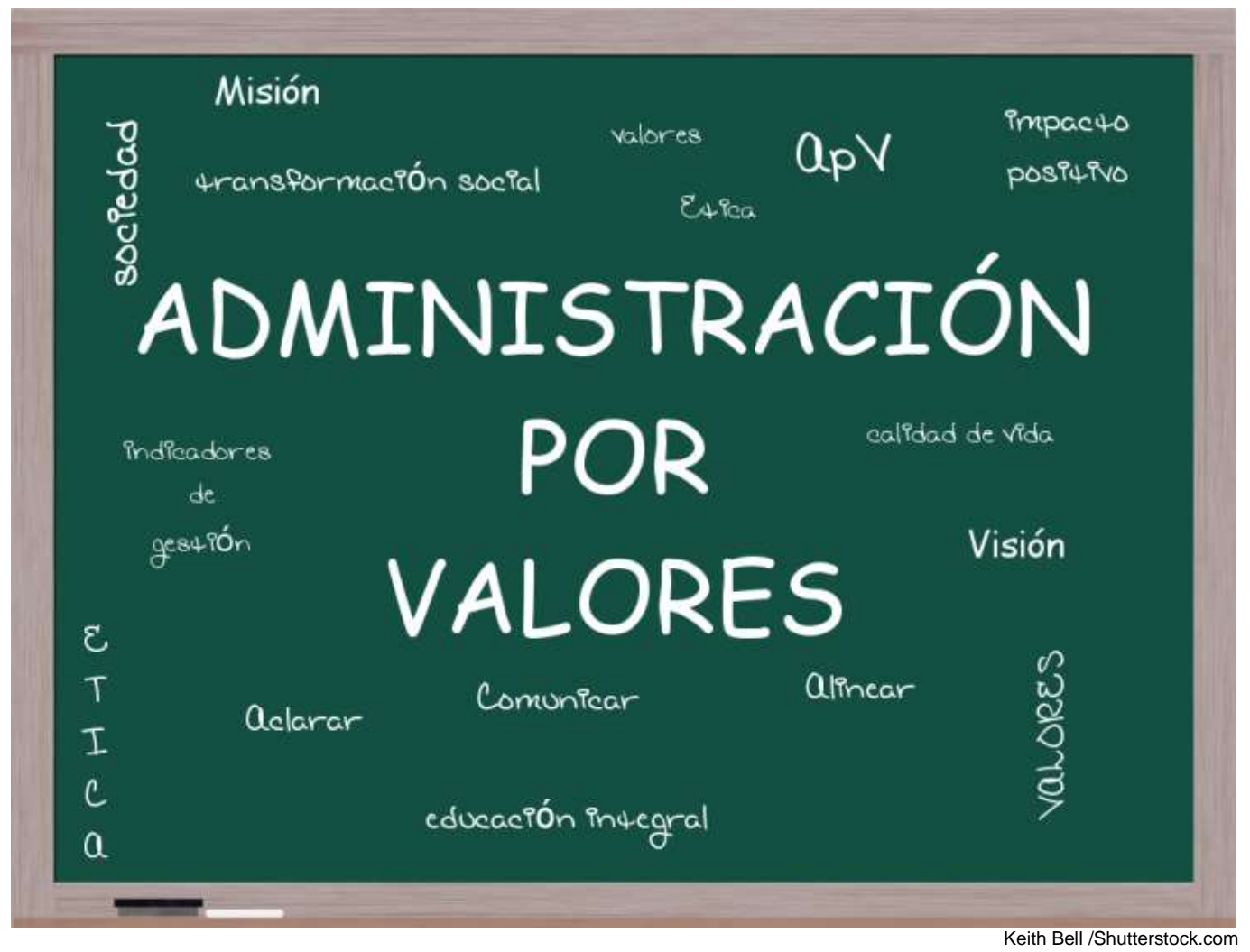

"Por encima del talento están los valores comunes: disciplina, amor, buena suerte, pero, sobre todo, tenacidad." James Baldwin (1924-1987)

\section{RESUMEN}

La Universidad de La Salle de Bogotá - Colombia tiene como Misión “...la educación integral y la generación de conocimiento que aporte a la transformación social y productiva del país...” (Proyecto Educativo Universitario Lasallista PEUL, 2007); partiendo de esta Misión al interior de la Facultad de Ciencias Administrativas y Contables se propone un modelo de Gestión Gerencial Académico/Administrativo, basada en la Administración Por Valores - ApV.

La investigación de la cual surge el presente documento, tiene por objetivo realizar una visión prospectiva de mejoramiento de la Facultad de Ciencias Administrativas y Contables de la Universidad de La Salle, al implementar un modelo de Administración por Valores - ApV en su Alta Gerencia (Directivos), como piedra angular del desarrollo del personal asociado a la Facultad y los estudiantes que se forman en ella.

La metodología se centró en la realización de una investigación descriptiva en una Facultad de Ciencias Administrativas y Contables de la ciudad de Bogotá - Colombia, mediante la recolección de información, a través de la observación directa y reconocimiento explorativo. 
Con base en lo anterior, el modelo de Administración por Valores podría impulsar los desarrollos Académico/Administrativos de la Facultad, al implementarse en primera instancia en la Alta Gerencia (Directivos de la Facultad: Decano, Secretario Académico, Directores de Programa, Asistentes Académicos, Coordinación Administrativa); se considera que al centrarse la primera etapa de implementación en la Alta Gerencia (Directivos), éstos pueden ser elementos multiplicadores del modelo aprendido en las demás dependencias de la Facultad, logrando una "Visión Compartida" del mismo.

\section{PALABRAS CLAVES}

Administración por valores, valores, calidad de vida, ética e indicador

Código JEL: 13 M - Administración de empresas y economía de la empresa; Marketing; Contabilidad.

\section{Introducción}

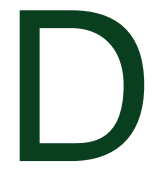

entro de las universidades en general el mejoramiento continuo es una de sus premisas, siendo su identidad, su misión, su visión y sus valores, los ejes rectores para su desarrollo, evolución y perfeccionamiento. De ésta forma, este artículo surge de una investigación en proceso de implementación en el interior de la Facultad de Ciencias Administrativas y Contables de la Universidad de La Salle, Bogotá - Colombia, donde se desea implementar el modelo de administración por valores - ApV aplicado a la alta gerencia (directivos) de esta facultad, propendiendo por su desarrollo y mejoramiento continuos.

Al implementar corrientes contemporáneas como la administración por valores se esperan los siguientes resultados posibles:

- Lograr un incremento en los indicadores de gestión frente los resultados de los tres (3) años inmediatamente anteriores.

- Mejorar la interacción entre los directivos de la facultad, y los demás actores que componen la misma, a través del desarrollo de los valores que la caracterizan y el desarrollo de una visión compartida.

- Incrementar la productividad de todas las unidades estratégicas de la Facultad de Ciencias Administrativas y Contables, mediante el compromiso de todos sus actores.

La investigación de la cual surge este artículo ofrece un aporte al conocimiento, al inferir que en el momento de aplicar el modelo de ApV, en una Facultad de Ciencias Administrativas y Contables, se podrían mejorar sus gestiones y evolucionar en el desarrollo de sus funciones sustantivas.

En el momento de generar una cultura basada en valores, se profundiza en el desarrollo organizacional y se propende por el desarrollo de una visión compartida a largo plazo, buscando ante todo un mejoramiento continuo que se vea reflejado en indicadores de gestión cada vez más positivos.

\section{Revisión conceptual}

Es necesario conocer de primera mano algunos conceptos básicos del modelo de administración por valores para lograr identificar la forma de implementación en la Facultad de Ciencias Administrativas y Contables de la Universidad de La Salle. En primera instancia, la administración por valores es la adopción de una filosofía de gestión, moral y empresarial acorde a una línea de pensamiento claramente definida. Busca que los integrantes de la organización se comprometan y actúen de manera coordinada y coherente respecto a la filosofía organizacional. (Blanchard Ken, O`Connor Michael, Administración por Valores, Norma, 2000.). De esta forma, cuando se crea una cultura basada en valores se busca la capacidad de supervivencia de la organización en el largo plazo sobre la base de una cultura fuerte en valores y en mejoramiento continuo. (García Salvador, Dolan Shimon; La Dirección por Valores.) 
La administración por valores - ApV implica, entre otros elementos,

- Generar mayor cohesión interna donde el compromiso es factor fundamental.

- Generar una mejor imagen externa, gracias a las relaciones basadas en la confianza a largo plazo.

- Aumentar el manejo ético en todos los niveles de la organización, disminuyendo las pérdidas, hechos deshonestos, robos o fraudes.

- Facilitar la selección de personal, gracias a perfiles éticos.

- Desarrollar una cadena de liderazgo confiable y comprometida con la filosofía de la organización.

De esta forma se puede concluir que la administración por valores, en general, busca mejorar la calidad de vida, tanto de la organización como de los individuos, mediante el ejercicio de confianza, responsabilidad y compromiso.

Una de las finalidades de esta investigación es rediseñar la cultura organizacional de la Facultad de Ciencias Administrativas y Contables de la Universidad de La Salle. En tal sentido, la administración por valores - $\mathrm{ApV}$, es un modelo valioso para tal fin, ya que está directamente orientada a rediseñar culturas y facilitar, el gobierno de los cambios estratégicos de la organización para adaptarse a su entorno y para superar sus tensiones internas. Esto se realiza mediante cuatro (4) aspectos fundamentales: (Reynaud Arana Camilo, La Administración por Valores una Alternativa para la Responsabilidad Social de las Empresas, 2009)

1. Los niveles de cultura de la organización.

2. La formulación de la Visión y la optimización de la cultura organizativa.
3. La "gestión del cambio" para cubrir la distancia entre la Visión de futuro y la realidad cultural.

4. Los valores para encauzar procesos hacia la visión estratégica.

Proceso para la implementación de la Administración por Valores - ApV en el interior de la Facultad de Ciencias

Administrativas y Contables de la Universidad de La Salle.

En el momento de implementar el modelo de administración por valores $-\mathrm{ApV}$, al interior de la Facultad de Ciencias Administrativas y Contables de la Universidad de La Salle es necesario tener en cuenta que la administración por valores infiere desarrollar nuevas dinámicas en cuanto a las decisiones gerenciales que se tomen, en función de generar una visión compartida de mediano y largo plazo.

Con base en lo anterior, el modelo de administración por valores $-\mathrm{ApV}$, propone las siguientes fases para su desarrollo:

\section{Cuadro 1. Fases del proceso de administración por valores.}

\begin{tabular}{|l|l|}
\hline Fase & Acción \\
\hline I & $\begin{array}{l}\text { Aclarar los valores, el propósito y la } \\
\text { visión. }\end{array}$ \\
\hline II & $\begin{array}{l}\text { Comunicar la visión y los valores } \\
\text { propios. }\end{array}$ \\
\hline III & $\begin{array}{l}\text { Alinear las prácticas propias diarias } \\
\text { con la visión y los valores propios. }\end{array}$ \\
\hline
\end{tabular}

Fuente: Administración por valores, Blanchard Ken, O`Connor Michael.

Fase I. Aclarar los valores, propósito y visión. (Blanchard Ken, O`Connor Michael, Administración por Valores, Norma, 2000.)

Para aclarar la visión y los valores, en primera instancia hay que obtener la aprobación de las directivas de la Facultad de Ciencias 
Administrativas y Contables. Una vez se obtiene, hay que decidir cuáles valores se quieren para impulsar la estrategia académico/administrativa, en un respectivo orden de prioridad.

Se debe igualmente definir la visión de la facultad, y determinar si ella refleja los valores que se han identificado. De no ser así, se procederá a revisarla para enfocarla de acuerdo con los valores.

La declaración de valores debe estar de acuerdo con la visión de la Universidad de La Salle. Hay que asegurarse que los valores sean aquellos por los cuales se anhela sea conocida y opere la facultad; cuanto más sencillos, directos y fáciles de entender sean, mayor será el nivel de aceptación y asimilación dentro de la organización.

Una vez que se tenga clara la versión de declaración de valores, se debe comunicar a las directivas de la facultad, para buscar la participación activa de estos en función de conocer los valores que ellos consideran prioritarios. Cuando se tenga esta lista de valores se compara con la primera declaración y se realizan los ajustes necesarios para articular las dos declaraciones.

Luego de articulado el grupo de directivos de la facultad con la declaración primaria de visión y valores en su respectivo orden de prioridad, es el momento de difundir la información para captar las opiniones de los demás actores de la facultad (profesores, estudiantes, egresados, pares, entre otros).

Finalmente, después de obtener todos los aportes antes mencionados, se sintetizan y se presentan a las directivas de la facultad, en función de obtener su aceptación y apoyo en el momento clave de implementación como tal de la administración por valores dentro de la facultad.

\section{Fase II. Comunicar la visión y los valores propios.}

Para comunicar la visión y valores en forma que se ajuste a agendas reales de trabajo de la facultad, es necesario iniciar el proceso de información convocando a reuniones de lanzamiento, donde se explique y aclare cada una de las siete etapas trabajadas para aclarar los valores, propósito y visión.

Una vez finalizada la socialización, se recomienda entregar a los diferentes actores de la facultad una copia laminada de la declaración de visión, los valores de la facultad; de este manera se logra fácil acceso a los modelos rectores y normativos de la Facultad y, a su vez, se pueden aplicar correctamente en el momento de toma de decisiones en cualquier situación que se requiera.

Además de la socialización anterior, se podría incrementar el nivel de conocimiento y apropiación de esta información por medio de la colocación de carteles y tarjetas de escritorio en los puntos de mayor acceso visual y mayor volumen de paso de los actores de la facultad, con el fin de asegurar mayor conciencia de la visión y valores. De esta manera, funciona como herramientas formativas de la actitud y conducta organizacional.

Fase III. Alinear las prácticas propias diarias con la visión y valores propios.

La alineación es la conjunción de los esfuerzos de la administración por valores $(\mathrm{ApV})$, una vez aclarados los valores y la visión, y se han comunicado a todos los interesados de la facultad, es el momento propicio para concentrarse en las prácticas y las conductas organizacionales para asegurar que sean compatibles con las intenciones declaradas, las prioridades y las correspondientes metas de rendimiento.

Para alinear las prácticas administrativas 
con los valores es necesario facilitar una "reducción de vacíos", que se refiere exactamente a eliminar situaciones que se presentan cuando una persona o un proceso está desalineado con lo que proponen los valores primarios.

Para ello, una solución propuesta por la administración por valores es la interacción con un "ombudsman" (se refiere a un defensor o mediador imparcial, Blanchard Ken, O`Connor Michael, administración por valores,

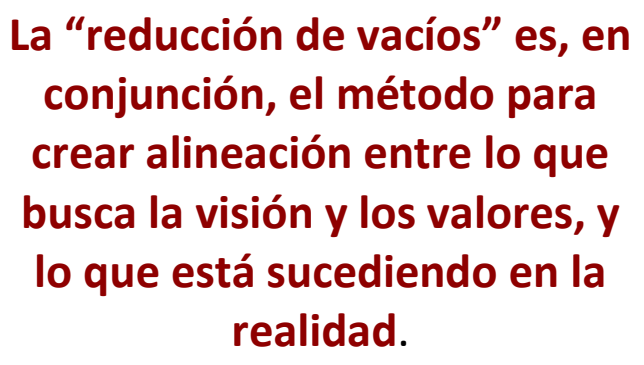

\section{Implementación final de la administración} por valores

Una vez se tienen los cimientos de la administración por valores, es necesario aplicarlo a cabalidad en la facultad. Esto se logra mediante una aclaración de la visión y los valores de la facultad que incluye obtener la aceptación de estos valores por parte de las principales partes interesadas.
Norma, 2000.) quien vela por la alineación de las personas y valores con los valores que son fundamentales para la facultad y ayuda de igual forma en las situaciones conflictivas cuando existe un problema potencial de alineación.

La "reducción de vacíos" es, en conjunción, el método para crear alineación entre lo que busca la visión y los valores, y lo que está sucediendo en la realidad. En función de lo anteriormente expresado, existen métodos de medición que se aplican coherentemente para determinar el nivel de alineación. (Cuadro 2)

Cuadro 2. Fase III. Métodos de medición de alineación en administración por valores (ApV).

\section{MÉTODOS DE MEDICIÓN}

Entrevistas de satisfacción de clientes y grupos de enfoque.

Evaluación administrativa y técnicas de retroalimentación.

Encuestas entre empleados (asociados) sobre las prácticas de la compañía.

Fuente: Administración por valores, Blanchard Ken, O`Connor Michael.
En seguida, para la fase de alineación se recomienda realizar una reunión con el equipo administrativo, para realizar un acercamiento en el cual probablemente se encontrará los siguientes tres (3) panoramas de personas: (Blanchard Ken, O`Connor Michael, Administración por valores, Norma, 2000.)

- Entusiastas inmediatos: Personas que prefieren el cambio y están dispuestos a aceptar la administración por valores tan pronto como reconocen los beneficios que ofrece para la facultad.

- Obstruccionistas: Los cuales presentan resistencia al cambio, actitud reacia a nuevas corrientes de cambio.

- Indecisos: Son quienes esperan para seguir a un líder, no tienen una actitud clara de decisión, es un grupo variable, este suele ser el grupo numérico más grande.

Antes de intentar abarcar demasiado dentro de la facultad en la alineación de la ApV, se recomienda empezar con un solo equipo de trabajo o unidad estratégica de "avanzada", donde existan algunos líderes entusiastas, los cuales faciliten la función de alineación.

Seguidamente se realiza la actividad de “articulación de valores y consenso". El propósito de esta actividad es ayudar a todos los 
individuos de un equipo o subgrupo a alinearse en torno a una serie de valores compartidos, para que resulte del ejercicio Valores con los cuales se puedan comprometer y que puedan usar como guías en el momento cuando toda la facultad funcione como unidad alrededor de la administración por valores - ApV.

\section{Metodología}

En el momento de realizar la investigación se implementó una metodología de investigación descriptiva en la Facultad de Ciencias Administrativas y Contables de la Universidad de La Salle, mediante la recolección de información a través de la observación directa y reconocimiento explorativo de la facultad.

Las herramientas principales que se utilizaron para la recolección de información fueron: evaluación de indicadores, observación directa; instrumentos que permitirán incrementar la confiabilidad de los datos. Como fuente primaria de investigación se utilizó la información recolectada y evaluada por el investigador dentro de Facultad de Ciencias Administrativas y Contables de la Universidad de La Salle.

En función de las fuentes secundarias, se utilizaron la información contenida en los documentos mencionados en la bibliografía, además de toda la información que enriqueció la investigación, en especial los documentos institucionales como: Proyecto Institucional de Desarrollo, Proyecto Educativo Universitario Lasallista y Enfoque Formativo Lasallista, entre otros.

\section{Resultados}

El resultado directo de la investigación fuente de la presente ponencia será la creación de la herramienta administrativa "administración por valores - ApV aplicada a la Facultad de Ciencias Administrativas y Contables de la Universidad de La Salle"
Se espera que la presente investigación sirva como una piedra angular para el evolucionado desarrollo de la administración por valores en el momento que se decida implementarlo; así como ofrecer a la Facultad de Ciencias Administrativas y Contables una solución administrativa para su gestión.

Al implementar la administración por valores en la Facultad de Ciencias Administrativas y Contables se busca generar un modo de gestión acorde a la filosofía de la facultad, mediante una participación activa de todas las partes productivas de la misma.

Es coherente pensar que en el momento de implementar la administración por valores en la facultad, los directivos de la misma, se encargará de ser elementos multiplicadores de esta corriente administrativa para todos los niveles de la facultad.

Se infiere que los indicadores de gestión de la Alta Gerencia (Directivos) de la Facultad de Ciencias Administrativas y Contables, tenderán a optimizarse, en buena medida, debido al mejoramiento de compromiso, respeto, espiritualidad y demás de valores que se hayan declarado como propios.

Como expectativa final se espera que los esfuerzos realizados sirvan para el mejoramiento de la calidad de vida, el nivel de entropía y valores aplicados al cristianismo que se profesan en la Universidad de La Salle y en la Facultad de Ciencias Administrativas y Contables.

\section{Conclusiones}

En el momento de implementarse el modelo de administración por valores - ApV en la Facultad de Ciencias Administrativas y Contables de la Universidad de La Salle, con base en sus lineamientos institucionales (Proyecto Institucional de Desarrollo, Proyecto Educativo Universitario Lasallista y Enfoque Formativo Lasallista, entre otros.), se lograría modular la fase I del modelo ApV (aclaración 
de los valores y visión aplicada a la Facultad de Ciencias Administrativas y Contables), generando así una visión compartida por todos los actores de la Facultad, en especial la alta gerencia de la misma.

De ésta forma, se lograría generar un mayor nivel de compromiso y seguimiento de los Valores y la Visión de la Facultad.

De implementarse el modelo de administración por valores $\mathrm{ApV}$, en el interior de la Facultad de Ciencias Administrativas y Contables y por medio de los lineamientos institucionales que ofrecen a la Universidad el marco normativo y moral de acción, se proyecta lograr encadenar la Fase I de Aclaración de Valores y Visión aplicada a la Facultad en mención; complementando así, el desarrollo de sus Valores recientemente declarados y enfocándolos al modelo ApV.

\section{Referencias bibliográficas}

Cornejo, Miguel Ángel, (2004). Valores de Excelencia para Triunfar, Grijalbo.

Cortina, Adela, (1996). El mundo de los Valores, Tecnos, Madrid.

Covey, Stephen, (1998). Liderazgo Centrado en Principios, Grijalbo.

Universidad de La Salle, (2008). Enfoque Formativo Lasallista - EFL.

Frederick, Robert, (2000). La Ética de los negocios, Oxford.

García Salvador \& Dolan Simón, (2003). La Dirección por Valores, McGraw Hill.

Universidad de La Salle, (2012). Hitos 14, Repensar la Académica Universitaria Lasallista.

Universidad de La Salle, (2010). Plan Institucional de Desarrollo 2010 - 2015.

Universidad de La Salle, (2007). Proyecto Educativo Universitario Lasallista - PEUL.
Risieri, Frondizi, (1995). ¿Qué son los valores?, Fondo de Cultura Económica.

Sen, Amartya, (1989). Sobre Ética y Economía, Alianza.

Yarce, Jorge, (2001). Los Valores son una Ventaja Competitiva, Liderazgo.

\section{Información del autor}

Andrés Felipe Sánchez Díez, M.B.A

afsanchez@lasalle.edu.co

Administrador de Empresas y Especialista en Alta Gerencia, Master of Business Administration - M.B.A

Secretario Académico, Facultad de Ciencias Administrativas y Contables, Universidad de La Salle. 